\title{
COINCIDENCE AND COMMON FIXED POINT THEOREMS IN COMPACT HAUSDORFF SPACES
}

\author{
ZEQING LIU, HAIYAN GAO, SHIN MIN KANG, AND YONG SOO KIM
}

Received 27 April 2004 and in revised form 26 January 2005

The existence of coincidence and fixed points for continuous mappings in compact Hausdorff spaces is established. Some equivalent conditions of the existence of fixed and common fixed points for any continuous mapping and a pair of mappings in compact Hausdorff spaces are given, respectively. Our results extend, improve, and unify the corresponding results due to Jungck, Liu, and Singh and Rao.

\section{Introduction}

Jungck [1, 2, 3] established some fixed and common fixed point theorems for continuous commuting mappings and gave criterion of the existence of fixed points for $C_{g f}$ in compact metric spaces. Several researchers extended Jungck's results in various aspects. Park [9] obtained some fixed point theorems for $f$-contractive mappings in compact metric spaces. Singh and Rao [10] established fixed point theorems for semigroups of selfmappings in compact Hausdorff spaces. Leader [4] studied uniformly contractive fixed points in compact metric spaces. Liu $[5,6,7,8]$ extended a few results due to Jungck, Singh, and Rao, and showed some fixed and common fixed point theorems in compact metric spaces and compact Hausdorff spaces, respectively.

Motivated and inspired by the papers in $[1,2,3,4,5,6,7,8,9,10]$, in this paper, we prove coincidence and fixed point theorems for continuous mappings in compact Hausdorff spaces. Meanwhile, we establish some equivalent conditions of the existence of fixed and common fixed points for any continuous mapping and a pair of mappings in compact Hausdorff spaces. Our results extend, improve, and unify the corresponding results due to Jungck [3], Liu [7, 8], and Singh and Rao [10].

\section{Preliminaries}

Throughout this paper, we assume that $(X, \tau)$ is a compact Hausdorff space and $F: X \times$ $X \rightarrow[0, \infty)$ is a continuous and symmetric function such that $F(x, y)=0$ if and only if $x=$ $y$. $\mathbb{N}$ and $I$ denote the set of positive integers and the identity mapping on $X$, respectively. 
For any mapping $f: X \rightarrow X$ and subsets $A$ and $B$ of $X$, define

$$
\begin{gathered}
C_{f}=\{g: g: X \longrightarrow X \text { and } g f=f g\}, \\
H_{f}=\left\{g: g: X \longrightarrow X \text { and } g \bigcap_{n \in \mathbb{N}} f^{n} X \subseteq \bigcap_{n \in \mathbb{N}} f^{n} X\right\}, \\
A_{f}=\left\{g: g: X \longrightarrow X \text { and } g \bigcap_{n \in \mathbb{N}} f^{n} X=\bigcap_{n \in \mathbb{N}} f^{n} X\right\}, \\
\delta_{F}(A, B)=\sup \{F(x, y): x \in A \text { and } y \in B\}, \\
\delta_{F}(A)=\delta_{F}(A, A) .
\end{gathered}
$$

Clearly, $A_{f} \cup C_{f} \subseteq H_{f}$ and $\left\{f^{n}: n \in \mathbb{N}\right\} \cup\{I\} \subseteq C_{f}$. In order to obtain our results, we need the following lemma which is due to Liu [8].

Lemma 2.1 [8]. Let $f$ and $g:(X, \tau) \rightarrow(X, \tau)$ be mappings such that $f g$ is continuous. Let $A=\bigcap_{n \in \mathbb{N}}(f g)^{n} X$. Then $A$ is a nonempty compact subset of $X, A=f g A$, and $h A \subseteq A$ for $h \in C_{f g}$. Moreover, $A=f A=g A$ provided that $f \in C_{g}$.

\section{Main results}

Theorem 3.1. Let $f, g$, and $h:(X, \tau) \rightarrow(X, \tau)$ be continuous mappings satisfying $f \in A_{g f}$, $h \in C_{f} \cap C_{g}$, and

$$
F(f x, g y)>\inf \{F(t, p t), F(h t, p t), F(h t, p h t), F(h x, h y): t \in\{x, y\} \text { and } p \in\{f, g\}\}
$$

for any $x, y \in X$ with $f x \neq g y$. Then at least one of the following conditions holds:

(1) $f$ has a fixed point in $X$;

(2) $g$ has a fixed point in $X$;

(3) $f$ and $h$ have a coincidence point in $X$;

(4) $g$ and $h$ have a coincidence point in X.

Proof. Let $A=\bigcap_{n \in \mathbb{N}}(g f)^{n} X$. By Lemma 2.1, $A$ is compact and $A=f A=g A=g f A \neq \varnothing$. Since $f, g$, and $h$ are continuous and $A$ is compact, there exist $a, b, u, v \in A$ such that

$$
\begin{gathered}
F(a, f a)=\inf \{F(x, f x): x \in A\}, \\
F(b, g b)=\inf \{F(x, g x): x \in A\}, \\
F(h u, f u)=\inf \{F(h x, f x): x \in A\}, \\
F(h v, g v)=\inf \{F(h x, g x): x \in A\} .
\end{gathered}
$$

We next consider the following cases.

Case 1. Suppose that

$$
F(a, f a)=\min \{F(a, f a), F(b, g b), F(h u, f u), F(h v, g v)\}
$$


By $g A=A$, there exists some $d \in A$ such that $g d=a$. Suppose that $f g d \neq g d$, that is, $f a \neq a$. From (3.1) and (3.3), we obtain that

$$
\begin{aligned}
F(f g d, g d) & >\inf \{F(t, p t), F(h t, p t), F(h t, p h t), F(h g d, h d): t \in\{g d, d\} \text { and } p \in\{f, g\}\} \\
& \geq \inf \{F(a, f a), F(b, g b), F(h u, f u), F(h v, g v)\} \\
& \geq F(a, f a),
\end{aligned}
$$

which implies that

$$
F(a, f a)=F(g d, f g d)=F(f g d, g d)>F(a, f a),
$$

which is a contradiction. Hence $a=f a$.

Case 2. Suppose that

$$
F(h u, f u)=\min \{F(a, f a), F(b, g b), F(h u, f u), F(h v, g v)\}
$$

By $g A=A$, there exists some $w \in A$ such that $g w=u$. Suppose that $f g w \neq g h w$. That is, $f u \neq h u$. From (3.1), (3.2), and (3.6), we infer that

$$
\begin{aligned}
F(f g w, g h w)> & \inf \{F(t, p t), F(h t, p t), F(h t, p h t), F(h g w, h h w): \\
& t \in\{g w, h w\} \text { and } p \in\{f, g\}\} \\
\geq & \inf \{F(a, f a), F(b, g b), F(h u, f u), F(h v, g v)\} \\
\geq & F(h u, f u),
\end{aligned}
$$

which means that

$$
F(h u, f u)=F(f g w, h g w)=F(f g w, g h w)>F(h u, f u)
$$

which is impossible. That is, $h u=f u$.

Case 3. Suppose that

$$
F(b, g b)=\min \{F(a, f a), F(b, g b), F(h u, f u), F(h v, g v)\}
$$

or

$$
F(h v, g v)=\min \{F(a, f a), F(b, g b), F(h u, f u), F(h v, g v)\}
$$

As in the proof of Case 1 or Case 2, we can conclude that (3) or (4) holds. This completes the proof.

Now we provide a few examples to illustrate Theorem 3.1.

Example 3.2. Let $X=[0,1] \cup[3,4]$ with the usual metric and $F: X \times X \rightarrow[0, \infty)$ by

$$
F(x, y)=\max \{x, y\}-\min \{x, y\} \quad \forall x, y \in X .
$$


Define $f, g, h: X \rightarrow X$ by

$$
\begin{aligned}
& f x= \begin{cases}x-x^{2} & \text { for } x \in[0,1], \\
x^{2}-6 x+12 & \text { for } x \in[3,4],\end{cases} \\
& g x= \begin{cases}x(1-x)\left(1-x+x^{2}\right) & \text { for } x \in[0,1], \\
x^{4}-12 x^{3}+54 x^{2}-108 x+84 & \text { for } x \in[3,4],\end{cases} \\
& h x= \begin{cases}0 & \text { for } x \in[0,1], \\
4 & \text { for } x \in[3,4] .\end{cases}
\end{aligned}
$$

It is easy to verify that for any $x, y \in X$ with $f x \neq g y$,

$$
\begin{aligned}
F(f x, g y)>0 & =F(h x, h f x)=F(h x, f h x) \\
& =\inf \{F(t, p t), F(h t, p t), F(h t, p h t), F(h x, h y): t \in\{x, y\} \text { and } p \in f, g\} .
\end{aligned}
$$

Clearly, the assumptions of Theorem 3.1 are fulfilled, and 0, 3, and 4 are common fixed points of $f$ and $g$, and 0,1 , and 4 are common coincidence points of $f, g$, and $h$.

Example 3.3. Let $X=\{1,2,3,4\}, \tau=\{A: A$ is a subset of $X\}$, and $F: X \times X \rightarrow[0, \infty)$ be defined by

$$
\begin{gathered}
F(x, x)=0, \quad F(x, y)=F(y, x) \quad \forall x, y \in X, \\
F(1,2)=F(2,3)=F(3,4)=1, \quad F(1,3)=F(2,4)=2, \quad F(1,4)=3 .
\end{gathered}
$$

Define $f, g$, and $h: X \rightarrow X$ by

$$
\begin{aligned}
& f 1=2, \quad f 2=3, \quad f 3=4, \quad f 4=3, \\
& g 1=3, \quad g 2=4, \quad g 3=3, \quad g 4=4 \text {, } \\
& h 1=4, \quad h 2=3, \quad h 3=4, \quad h 4=3 \text {. }
\end{aligned}
$$

Then $(X, \tau)$ is a compact Hausdorff space, $f, g$, and $h$ are continuous self-mappings of $X$,

$$
\begin{gathered}
\bigcap_{n \in \mathbb{N}}(g f)^{n} X=\{3,4\}=f \bigcap_{n \in \mathbb{N}}(g f)^{n} X, \quad h \in C_{f} \cap C_{g}, \\
F(f x, g y)>0=\inf \{F(t, p t), F(h t, p t), F(h t, p h t), F(h x, h y): \\
t \in\{x, y\} \text { and } p \in\{f, g\}\}
\end{gathered}
$$

for any $x, y \in X$ with $f x \neq g y$. Obviously, $f$ has no fixed point, $g$ and $h$ have no coincidence point, $f$ and $h$ have three coincidence points 2,3 , and 4 , and $g$ has two fixed points 3 and 4 . 
Example 3.4. Let $(X, \tau)$ be as in Example 3.2. Define $f, g$, and $h: X \rightarrow X$ by

$$
\begin{array}{ccrr}
f 1=4, & f 2=3, & f 3=2, & f 4=1, \\
g 1=2, & g 2=1, & g 3=4, & g 4=3, \\
& h x=g x & \forall x \in X . &
\end{array}
$$

Then $f, g$, and $h$ are continuous self-mappings of $(X, \tau)$,

$$
\begin{gathered}
\bigcap_{n \in \mathbb{N}}(g f)^{n} X=X=f \bigcap_{n \in \mathbb{N}}(g f)^{n} X, \quad h \in C_{f} \cap C_{g}, \\
F(f x, g y)>0=\inf \{F(t, p t), F(h t, p t), F(h t, p h t), F(h x, h y): \\
t \in\{x, y\} \text { and } p \in\{f, g\}\}
\end{gathered}
$$

for any $x, y \in X$ with $f x \neq g y$. Obviously, $f$ and $g$ have no fixed point, respectively, $f$ and $h$ have no coincidence point, while $g$ and $h$ have four coincidence points $1,2,3$, and 4 .

Remark 3.5. Examples 3.3 and 3.4 show that the conclusions of Theorem 3.1 may not hold simultaneously.

Theorem 3.6. Let $f, g$, and $h:(X, \tau) \rightarrow(X, \tau)$ be continuous mappings satisfying $f \in A_{g f}$, $h \in C_{f} \cap C_{g}$, and

$$
F(f x, g y)>\inf \{F(t, p t), F(h t, p t): t \in\{x, y\} \text { and } p \in\{f, g\}\}
$$

for any $x, y \in X$ with $f x \neq g y$. Then at least one of (1)-(4) holds.

Proof. Notice that (3.19) implies (3.1). Thus Theorem 3.6 follows from Theorem 3.1. This completes the proof.

Theorem 3.7. Let $f, g:(X, \tau) \rightarrow(X, \tau)$ be continuous mappings satisfying $f \in A_{g f}$ and

$$
F(f x, g y)>\inf \left\{F(h t, p h t), F(h x, h y): t \in\{x, y\}, p \in\{f, g\} \text { and } h \in C_{f} \cap C_{g}\right\}
$$

for any $x, y$ in $X$ with $f x \neq g y$. Then at least one of $f$ and $g$ has a fixed point.

The proof of Theorem 3.7 is now essentially the same as the proof of Theorem 3.1 and therefore is omitted.

Remark 3.8. Jungck [3, Theorem 3.4] and Liu [8, Theorem 3] are special cases of Theorem 3.7.

Theorem 3.9. Let $f:(X, \tau) \rightarrow(X, \tau)$ be a continuous mapping. Then the following statements are equivalent:

(5) $f$ has a fixed point;

(6) for any $x, y$ in $X$ with $f x \neq f y$, there exists $h \in C_{f}$ such that

$$
F(f x, f y)>F(h x, h y)
$$

(7) for any $x, y$ in $X$ with $f x \neq f y, f$ satisfies that

$$
F(f x, f y)>\inf \left\{F(x, f x), F(y, f y), F(h x, h y): h \in C_{f}\right\} ;
$$


(8) for any $x, y$ in $X$ with $f x \neq f y, f$ satisfies that

$$
F(f x, f y)>\inf \left\{F(h x, f h x), F(h y, f h y), F(h x, h y): h \in C_{f}\right\} .
$$

Proof. $(5) \Leftrightarrow(6)$ and $(5) \Leftrightarrow(7)$ are given in [8]. (7) $\Rightarrow(8)$ is clear. We now suppose that (8) holds. Taking $f=g$ in Theorem 3.7, we easily get that $f$ has a fixed point. That is, (8) implies (5). This completes the proof.

THEOREM 3.10. For a pair of self-mappings $f$ and $g$ of $(X, \tau)$, the following conditions are equivalent:

(9) $f$ and $g$ have a common fixed point;

(10) there exist $m, n \in \mathbb{N}$ and $s, t: X \rightarrow X$ such that $s, g, f \in A_{\text {tsg } f}$, tsg $f$ is continuous, and

$$
F\left(s^{m} x, t^{n} y\right)<\sup \left\{F(c u, b v): u, v \in\{x, y\} \text { and } c, b \in H_{t s g f}\right\}
$$

for any $x, y \in X$ with $s^{m} x \neq t^{n} y$;

(11) there exist $m, n \in \mathbb{N}$ and $h: X \rightarrow X$ such that $g, f \in A_{h g f}$, hg $f$ is continuous, and

$$
F\left(h^{m} x, h^{n} y\right)<\sup \left\{F(t u, s v): u, v \in\{x, y\} \text { and } t, s \in H_{h g f}\right\}
$$

for any $x, y \in X$ with $h^{m} x \neq h^{n} y$;

(12) there exist $m, n \in \mathbb{N}$ and $s, t: X \rightarrow X$ such that $f \in A_{s f}, g \in A_{t g}, s f$ and tg are continuous, and

$$
F\left(s^{m} x, t^{n} y\right)<\sup \left\{F(c x, b y): c \in H_{s f}, b \in H_{t g}\right\}
$$

for any $x, y \in X$ with $s^{m} x \neq t^{n} y$.

Proof. Suppose that $f \omega=g \omega=\omega$ for some $\omega \in X$. Define mappings $s, t$, and $h: X \rightarrow X$ by $s x=t x=h x=\omega$ for all $x \in X$. It is easy to show that (10), (11), and (12) hold. Let (10) hold. Define $B=\bigcap_{n \in \mathbb{N}}(\operatorname{tsg} f)^{n} X$. By Lemma 2.1, we get that $t \operatorname{sg} f B=B \neq \varnothing$. Note that $s B=g B=f B=B$. Then $t B=\operatorname{tsg} f B=B$. We now assert that $B$ is a singleton. Otherwise, $\delta_{F}(B)>0$. Since $B$ is compact, there exist $p, q \in B$ with $\delta_{F}(B)=F(p, q)$. From $s B=t B=$ $B$, we can find $x, y \in B$ such that $s^{m} x=p$ and $t^{n} y=q$. If $p \neq q$, then

$$
\delta_{F}(B)=F\left(s^{m} x, t^{n} y\right)<\sup \left\{F(c u, b v): u, v \in\{x, y\} \text { and } c, b \in H_{t s g f}\right\} \leq \delta_{F}(B),
$$

which is a contradiction. Hence $\delta_{F}(B)=0$. This implies that $B=\{\omega\}$ for some $\omega \in X$. Clearly, $\omega$ is a common fixed point of $f$ and $g$. Hence, (10) implies (9). Similarly we can show that (11) implies (9). We next prove that (12) implies (9). Let (12) hold. Define $C=\bigcap_{n \in \mathbb{N}}(s f)^{n} X$ and $D=\bigcap_{n \in \mathbb{N}}(t g)^{n} X$. It follows from Lemma 2.1 that $C$ and $D$ are nonempty compact subsets of $X, c C \subseteq C$ for $c \in H_{s f}$, and $b D \subseteq D$ for $b \in H_{t g}$. Note that $f C=C$ and $g D=D$. Then $s C=s f C=C$ and $t D=\operatorname{tg} D=D$. Since $C$ and $D$ are compact and $F$ is continuous, there exist $p \in C$ and $q \in D$ such that $\delta_{F}(C, D)=F(p, q)$. From $s C=C$ and $t D=D$, we can find $x \in C$ and $y \in D$ such that $s^{m} x=p$ and $t^{n} y=q$. If $p \neq q$, then

$$
\delta_{F}(C, D)=F\left(s^{m} x, t^{n} y\right)<\sup \left\{F(c x, b y): c \in H_{s f}, b \in H_{t g}\right\} \leq \delta_{F}(C, D),
$$


which is a contradiction. Therefore $p=q$. This implies that $C=D=\{a\}$ for some $a \in X$. It is easy to see that $a=c a=b a$ for all $c \in H_{s f}$ and $b \in H_{t g}$; in particular, $a=f a=g a$. That is, (9) holds. This completes the proof.

Remark 3.11. Theorem 3.10 generalizes [7, Theorem 1] and [10, Theorem 7].

From Theorem 3.10, we have the following theorem.

Theorem 3.12. Let $f, g:(X, \tau) \rightarrow(X, \tau)$ be commuting mappings. Then (9) is equivalent to each of the following:

(13) there exist $m, n \in \mathbb{N}$ and $s, t: X \rightarrow X$ such that $s \in C_{t}, s, t \in C_{f} \cap C_{g}$, tsg $f$ is continuous, and

$$
F\left(s^{m} x, t^{n} y\right)<\sup \left\{F(c u, b v): u, v \in\{x, y\} \text { and } c, b \in C_{t s g f}\right\}
$$

for any $x, y \in X$ with $s^{m} x \neq t^{n} y$;

(14) there exist $m, n \in \mathbb{N}$ and $h: X \rightarrow X$ such that $h \in C_{f} \cap C_{g}$, $h g f$ is continuous, and

$$
F\left(h^{m} x, h^{n} y\right)<\sup \left\{F(t u, s v): u, v \in\{x, y\} \text { and } t, s \in C_{h g f}\right\}
$$

for any $x, y \in X$ with $h^{m} x \neq h^{n} y$

(15) there exist $m, n \in \mathbb{N}$ and $s, t: X \rightarrow X$ such that $s \in C_{f}, t \in C_{g}$, sf and tg are continuous, and

$$
F\left(s^{m} x, t^{n} y\right)<\sup \left\{F(c x, b y): c \in C_{s f}, b \in C_{t g}\right\}
$$

for any $x, y \in X$ with $s^{m} x \neq t^{n} y$.

Remark 3.13. The following simple example reveals that Theorem 3.12 extends properly [3, Theorem 4.2] and that the common fixed point of $f$ and $g$ in Theorem 3.12 may not be unique.

Example 3.14. Let $X=[0,1]$ with the usual metric $d$ and $F=d$. Define mappings $f, g$, and $v: X \rightarrow X$ by

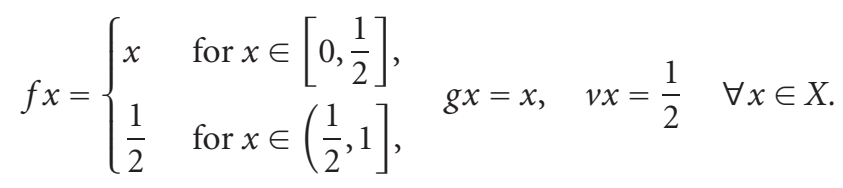

Let $s=t=v$ and $m=n=1$. Then (9) and (13) hold. Clearly, each $\omega \in[0,1 / 2]$ is a common fixed point of $f$ and $g$. But [3, Theorem 4.2] is not applicable because

$$
d(f 0, g 1)=1=\delta_{d}\left(\left\{h z: z \in\{0,1\} \text { and } h \in C_{g f}\right\}\right) .
$$

Theorem 3.15. Let $f:(X, \tau) \rightarrow(X, \tau)$ be a continuous mapping. Then $(5)$ is equivalent to each of the following statements:

(16) for any $x, y \in X$ with $x \neq y$, there exists $h \in C_{f}$ satisfying

$$
F(x, y)>F(h x, h y)
$$


852 Coincidence and common fixed point theorems

(17) for any $x, y \in X$ with $x \neq y, f$ satisfies that

$$
F(x, y)>\inf \left\{F(x, f x), F(y, f y), F(h x, h y): h \in C_{f}\right\} ;
$$

(18) for any $x, y \in X$ with $x \neq y, f$ satisfies that

$$
F(x, y)>\inf \left\{F(h x, f h x), F(h y, f h y), F(h x, h y): h \in C_{f}\right\} ;
$$

(19) there exists $h \in C_{f}$ such that

$$
F(h x, h y)<F(x, y)
$$

for any $x, y \in X$ with $x \neq y$;

(20) there exists $h \in C_{f}$ such that

$$
F(h x, h y)<F(f x, f y)
$$

for any $x, y \in X$ with $f x \neq f y$;

(21) there exist $m, n \in \mathbb{N}$ and $h \in C_{f}$ such that $h f$ is continuous, and

$$
F\left(h^{m} x, h^{n} y\right)<\sup \left\{F(c u, b v): u, v \in\{x, y\} \text { and } c, b \in H_{h f}\right\}
$$

for any $x, y \in X$ with $h^{m} x \neq h^{n} y$;

(22) there exist $m, n \in \mathbb{N}$ and $h: X \rightarrow X$ such that $f \in A_{h f}$, $h f$ is continuous, and

$$
F\left(h^{m} x, h^{n} y\right)<\sup \left\{F(c u, b v): u, v \in\{x, y\} \text { and } c, b \in H_{h f}\right\}
$$

for any $x, y \in X$ with $h^{m} x \neq h^{n} y$;

(23) there exist $m, n \in \mathbb{N}$ and $s, t \in C_{f}$ such that $s f$ and $t f$ are continuous, and

$$
F\left(s^{m} x, t^{n} y\right)<\sup \left\{F(c x, b y): c \in H_{s f}, b \in H_{t f}\right\}
$$

for any $x, y \in X$ with $s^{m} x \neq t^{n} y$;

(24) there exist $m, n \in \mathbb{N}$ and $s, t: X \rightarrow X$ such that $f \in A_{s f} \cap A_{t f}$, $s f$ and $t f$ are continuous, and

$$
F\left(s^{m} x, t^{n} y\right)<\sup \left\{F(c x, b y): c \in H_{s f}, b \in H_{t f}\right\}
$$

for any $x, y \in X$ with $s^{m} x \neq t^{n} y$.

Proof. In view of Theorems 3.9 and 3.10, we conclude easily that $(19) \Rightarrow(16) \Rightarrow(17) \Rightarrow(18)$, $(20) \Rightarrow(6) \Rightarrow(5),(21) \Rightarrow(22) \Rightarrow(11) \Rightarrow(9) \Rightarrow(5)$ with $g=I$, and $(23) \Rightarrow(24) \Rightarrow(12) \Rightarrow(9) \Rightarrow(5)$ with $g=f$. Let (5) hold and let $w$ be a fixed point of $f$. Define $h x=s x=t x=w$ for all $x \in X$. It is easy to see that (19), (20), (21), and (23) hold. In order to finish the proof, we need to prove that $(18) \Rightarrow(5)$. Now assume that (18) holds. Let $A=\bigcap_{n \in \mathbb{N}} f^{n} X$. By Lemma 2.1, $f A=A \neq \varnothing$ and $A$ is compact. By the continuity of $f$ and the compactness of $A$, there exists some $a \in A$ such that

$$
F(a, f a)=\inf \{F(x, f x): x \in A\} .
$$


Since $f A=A$, there exists $y \in A$ such that $f a=y$. Suppose that $a \neq f a$. Then

$$
F(a, f a)>\inf \left\{F(h a, f h a), F(h f a, f h f a), F(h a, h f a): h \in C_{f}\right\} \geq F(a, f a),
$$

which is impossible. Hence $a$ is a fixed point of $f$ and (5) holds. This completes the proof.

\section{Acknowledgments}

The authors would like to thank the referees for their helpful suggestions towards the improvement of this paper. This work was supported by the Science Research Foundation of Educational Department of Liaoning Province (2004C063) and Korea Research Foundation Grant (KRF-2003-005-C00013).

\section{References}

[1] G. Jungck, Commuting mappings and fixed points, Amer. Math. Monthly 83 (1976), no. 4, 261263.

[2] , Periodic and fixed points, and commuting mappings, Proc. Amer. Math. Soc. 76 (1979), no. 2, 333-338.

[3] Common fixed points for commuting and compatible maps on compacta, Proc. Amer. Math. Soc. 103 (1988), no. 3, 977-983.

[4] S. Leader, Uniformly contractive fixed points in compact metric spaces, Proc. Amer. Math. Soc. 86 (1982), no. 1, 153-158.

[5] Z. Q. Liu, A note on fixed point in compact metric spaces, Indian J. Math. 34 (1992), no. 2, 173-176.

[6] Common fixed point theorems in compact metric spaces, Pure Appl. Math. Sci. 37 (1993), no. 1-2, 83-87.

[7] Characterizations of fixed and common fixed points, Acta Sci. Math. (Szeged) 59 (1994), no. 3-4, 579-584.

[8] Some fixed point theorems in compact Hausdorff spaces, Indian J. Math. 36 (1994), no. 3, 235-239.

[9] S. Park, Fixed points of $f$-contractive maps, Rocky Mountain J. Math. 8 (1978), no. 4, 743-750.

[10] S. L. Singh and K. P. R. Rao, Coincidence and fixed points for four mappings, Indian J. Math. 31 (1989), no. 3, 215-223.

Zeqing Liu: Department of Mathematics, Liaoning Normal University, P.O. Box 200, Dalian, Liaoning 116029, China

E-mail address: zeqingliu@dl.cn

Haiyan Gao: Department of Mathematics, Liaoning Normal University, P.O. Box 200, Dalian, Liaoning 116029, China

E-mail address: haiyangao@mail.china.com

Shin Min Kang: Department of Mathematics, Gyeongsang National University, Chinju 660-701, Korea

E-mail address: smkang@nongae.gsnu.ac.kr

Yong Soo Kim: Department of Mathematics and Research Institute of Natural Science, Gyeongsang National University, Chinju 660-701, Korea

E-mail address: math@nongae.gsnu.ac.kr 


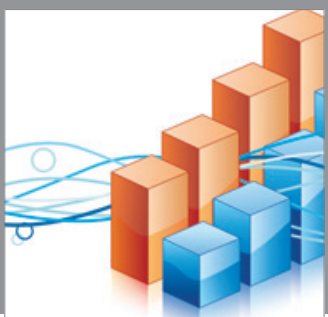

Advances in

Operations Research

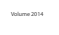

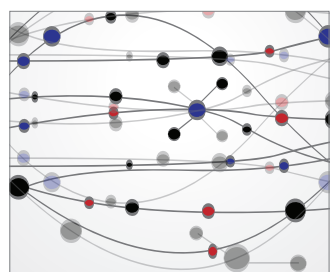

\section{The Scientific} World Journal
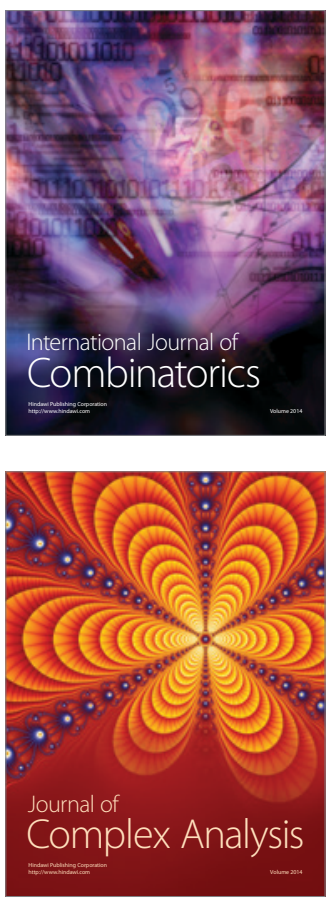

International Journal of

Mathematics and

Mathematical

Sciences
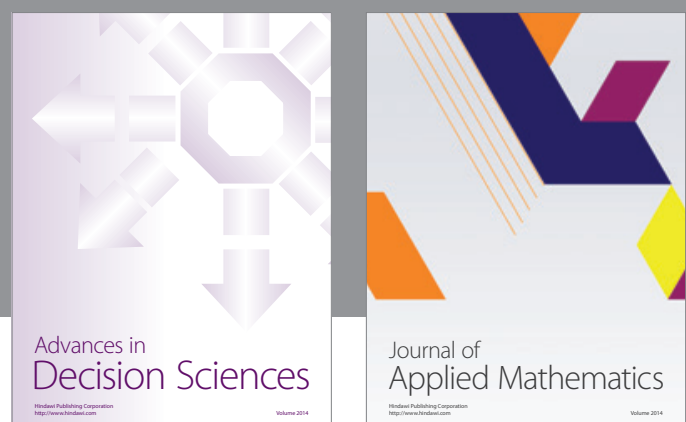

Journal of

Applied Mathematics
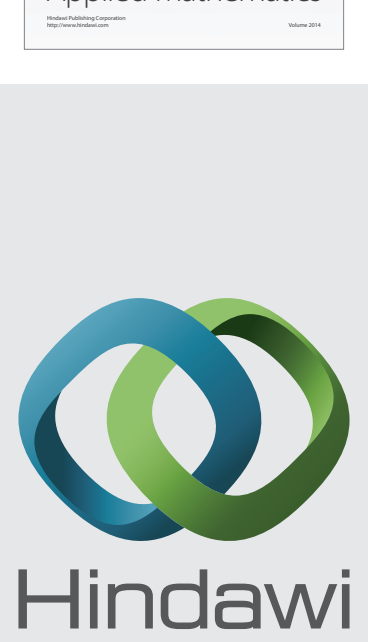

Submit your manuscripts at http://www.hindawi.com
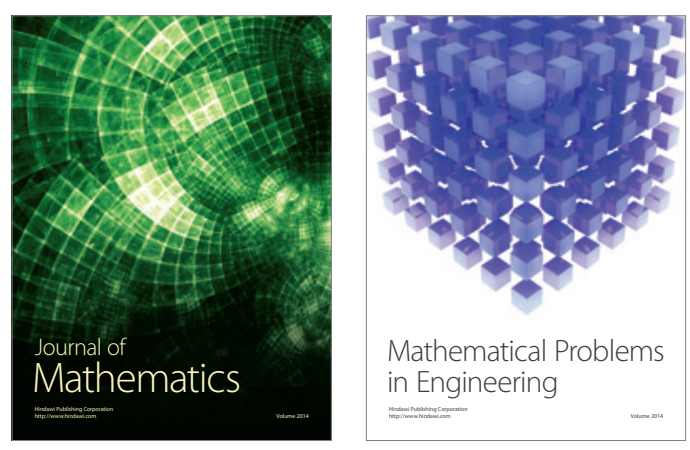

Mathematical Problems in Engineering
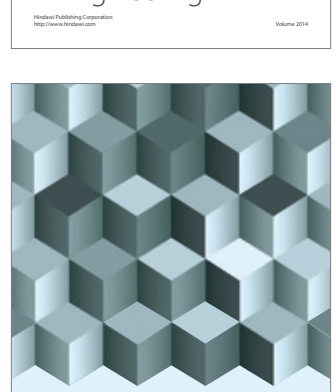

Journal of

Function Spaces
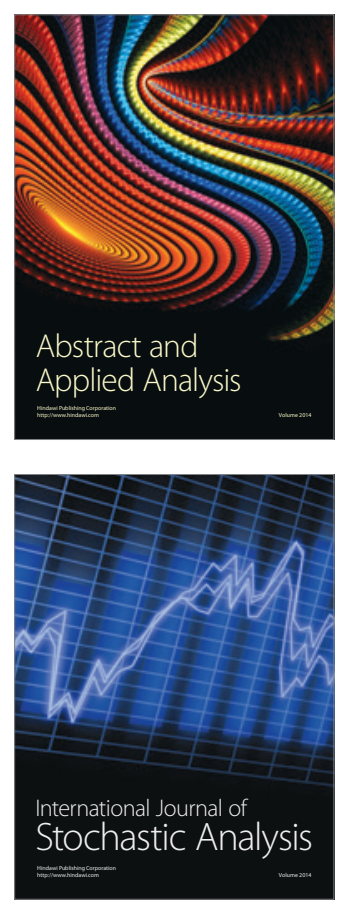

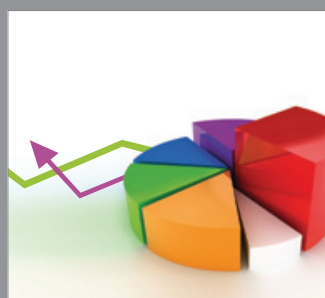

ournal of

Probability and Statistics

Promensencen
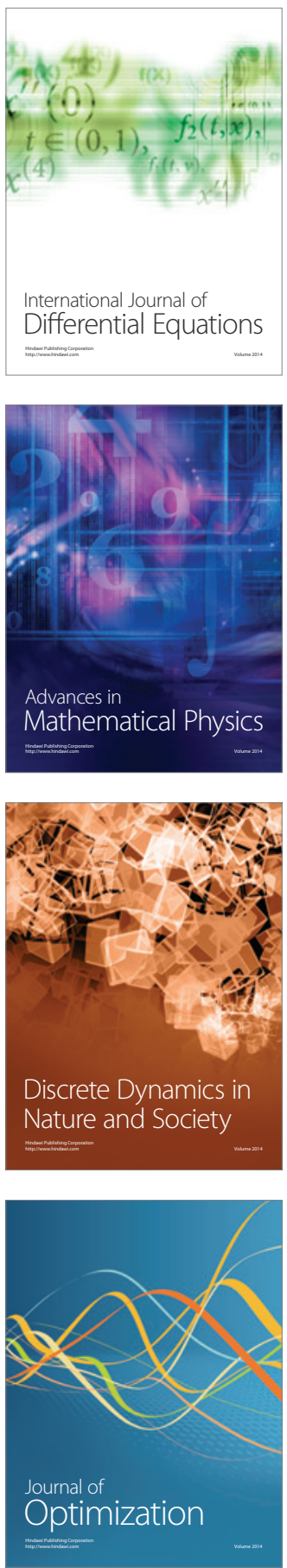